\title{
Localization algorithms for multilateration (MLAT) systems in airport surface surveillance
}

\section{Ivan A. Mantilla-Gaviria, Mauro Leonardi, Gaspare Galati \& Juan V. Balbastre-Tejedor}

Signal, Image and Video Processing

ISSN 1863-1703

SIViP

DOI 10.1007/s11760-013-0608-1

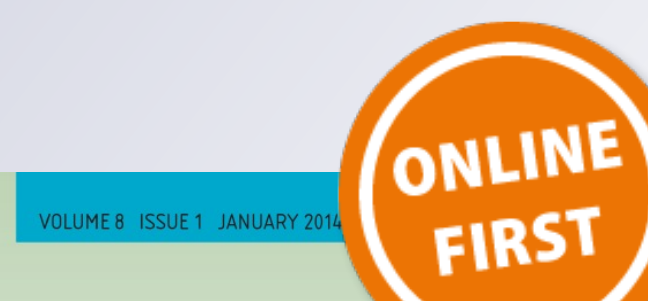

Signal, Image

and Video

Processing 
Your article is protected by copyright and all rights are held exclusively by SpringerVerlag London. This e-offprint is for personal use only and shall not be self-archived in electronic repositories. If you wish to self-archive your article, please use the accepted manuscript version for posting on your own website. You may further deposit the accepted manuscript version in any repository, provided it is only made publicly available 12 months after official publication or later and provided acknowledgement is given to the original source of publication and a link is inserted to the published article on Springer's website. The link must be accompanied by the following text: "The final publication is available at link.springer.com". 


\title{
Localization algorithms for multilateration (MLAT) systems in airport surface surveillance
}

\author{
Ivan A. Mantilla-Gaviria • Mauro Leonardi • \\ Gaspare Galati · Juan V. Balbastre-Tejedor
}

Received: 16 May 2013 / Revised: 11 December 2013 / Accepted: 25 December 2013

(C) Springer-Verlag London 2014

\begin{abstract}
We present a general scheme for analyzing the performance of a generic localization algorithm for multilateration (MLAT) systems (or for other distributed sensor, passive localization technology). MLAT systems are used for airport surface surveillance and are based on time difference of arrival measurements of Mode S signals (replies and $1,090 \mathrm{MHz}$ extended squitter, or 1090ES). In the paper, we propose to consider a localization algorithm as composed of two components: a data model and a numerical method, both being properly defined and described. In this way, the performance of the localization algorithm can be related to the proper combination of statistical and numerical performances. We present and review a set of data models and numerical methods that can describe most localization algorithms. We also select a set of existing localization algorithms that can be considered as the most relevant, and we describe them under the proposed classification. We show that the performance of any localization algorithm has two components, i.e., a statistical one and a numerical one. The statistical performance is related to providing unbiased and minimum variance solutions, while the numerical one is related to ensuring the convergence of the solution. Furthermore, we show that a robust localization (i.e., statistically and numer-
\end{abstract}

I. A. Mantilla-Gaviria · J. V. Balbastre-Tejedor

Instituto ITACA, Universidad Politécnica de Valencia,

Camino de Vera S/N, 46022 Edificio 8G, Acceso B,

Valencia, Spain

e-mail: iamantillagaviria@gmail.com

J. V. Balbastre-Tejedor

e-mail: jbalbast@itaca.upv.es

M. Leonardi · G. Galati ( $\square)$

DIE, Tor Vergata University, Via del Politecnico 1, 00133 Rome, Italy

e-mail: gaspare.galati@uniroma2.it; gaspare.galati@gmail.com

M. Leonardi

e-mail: mauro.leonardi@uniroma2.it ically efficient) strategy, for airport surface surveillance, has to be composed of two specific kind of algorithms. Finally, an accuracy analysis, by using real data, is performed for the analyzed algorithms; some general guidelines are drawn and conclusions are provided.

Keywords Localization algorithms - Multilateration . Time difference of arrival - Airport surface surveillance . Air traffic control

\section{Introduction}

A Multilateration (MLAT) system detects, locates, and identifies cooperating targets by receiving and processing suitable signals emitted by on-board transponder devices, according to the Secondary Surveillance Radar (SSR) international standards (e.g., the Mode A/C and Mode S signals). The complete description of such systems is beyond the scope of this paper, and the interested reader can refer to the international standards [1,2] and to the technical literature [3-5]. These systems were initially conceived for airport traffic surveillance, including both aircrafts and some surface vehicles carrying onboard equivalent equipment called "non-transponder device." Later, they were extended to air traffic around one or more airports, with the name of Wide Area Multilateration (WAM).

In MLAT systems, receiving stations are placed in some strategic locations around the area to be covered. The system uses the Mode A/C- and Mode S-based transmissions, i.e., the spontaneous Mode $\mathrm{S}$ squitter, the asynchronous transponder replies, as well as the responses to interrogations elicited by the system itself. The received signals are sent to the Central Processing Subsystem (CPS) where the transponder (i.e., the target) position is calculated $[1,2]$. In the standard ver- 


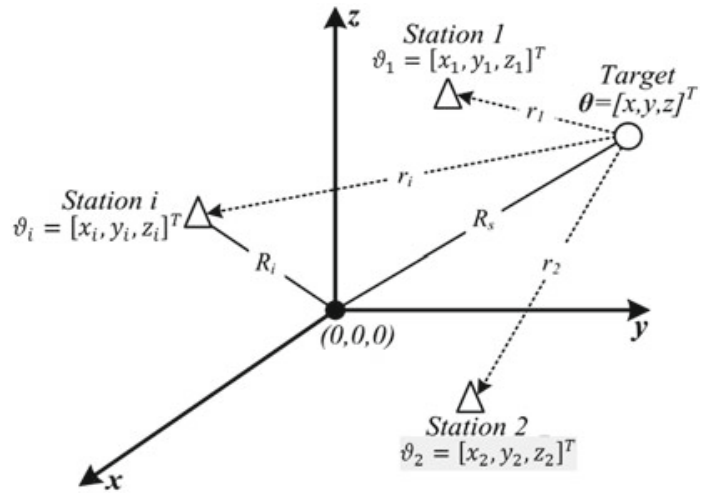

Fig. 1 MLAT system description

sion of MLAT systems, the localization is based on the time difference of arrival (TDOA) principle, as shown in Fig. 1.

The TDOA principle consists in relating the unknown target position, i.e., $\boldsymbol{\theta}=[x, y, z]^{T}$, being $T$ the transpose operator, with a set of known parameters, i.e., the TDOA measurements. They are the time of arrival (TOA) differences between the $i$ th receiving station and a reference one (normally designated as the number 1). The resulting function geometrically represents an hyperboloid, and it can be expressed as follows:

$$
\begin{aligned}
& \widehat{\mathrm{TDOA}}_{i, 1}(\boldsymbol{\theta})=\frac{\mathbf{1}}{\boldsymbol{c}}\left(\left\|\boldsymbol{\theta}-\boldsymbol{\vartheta}_{\boldsymbol{i}}\right\|-\left\|\boldsymbol{\theta}-\boldsymbol{\vartheta}_{1}\right\|\right)+n_{i, 1}, \\
& i=2, \ldots, N_{S}
\end{aligned}
$$

where the superscript denotes that is an estimated quantity, $\boldsymbol{\vartheta}_{i}=\left[x_{i}, y_{i}, z_{i}\right]^{T}$ is the $i$ th station position, $n_{i, 1}$ is a TDOA random noise term, which generally is assumed to be zero mean Gaussian distributed [6], $c$ is the speed of light, and $N_{s}$ is the total number of receiving stations. The TDOA measurement (estimated) in (1) can be also defined as the range difference measurement as follows:

$\widehat{m}_{i, 1}(\boldsymbol{\theta})=c \widehat{\mathrm{TDOA}}_{i, 1}=\left(r_{i}-r_{1}\right)+n_{i, 1}$,

where $r_{i}=\left\|\boldsymbol{\theta}-\boldsymbol{\vartheta}_{i}\right\|$. Finally, in order to obtain a numerical data concerning $\boldsymbol{\theta}$, a localization algorithm manipulates the set of measurements in the form of (2) to construct and to solve a system of equations, whose solution is an inverse problem that can be generally expressed as follows:

$\mathbf{G} \boldsymbol{\theta}+\mathbf{n}=\mathbf{m}$,

where $\mathbf{G}$ is the coefficient matrix of the resulting inverse problem, $\mathbf{n}$ is the measurement additive noise vector, and $\mathbf{m}$ is the noiseless range differences vector. Thus, the aim of a localization algorithm is to set both the matrix $\mathbf{G}$ and the unknown transponder position vector $\boldsymbol{\theta}$ and to obtain a numerical value for $\boldsymbol{\theta}$ that fits (3), under the additive noise $\mathbf{n}$.

It is well known that the position accuracy of MLAT systems depends on three elements, namely (1) the measure- ments accuracy, (2) the spatial distribution of the stations (also called system geometry) that is quantified by the Dilution of precision (DOP) parameter, and (3) the localization algorithm used to convert the TOA/TDOA measurements space [i.e., the set of measurements in (1)] into the position one, referenced to the Cartesian space $(x, y, z)$ [7-9]. It is generally assumed that the MLAT position errors can be modeled by an unbiased Gaussian distribution with a given variance, where the measurements accuracy and the geometrical effects (i.e., as quantified by the DOP) set its minimum variance values [9]. Nevertheless, this limit only represents the best case for the system accuracy that can be achieved with the corresponding measurements and system geometry. In this sense, the fact that the operational system (regardless of the hardware implementation quality) reaches or not this limit depends on the overall efficiency of the localization algorithm.

Generally, the localization algorithms are classified into two families: (1) open form (sometimes also called iterative) and (2) closed form (sometimes also called direct). In this classification, a localization algorithm is an entity that reconstructs the target position, given a set of TDOA measurements. The interested reader may see $[7,10,11]$ and the references therein for some guidelines in MLAT systems, and [12] for an equivalent comparison for Global Positioning System (GPS) geolocation algorithms. However, really (as we describe later), a localization algorithm is composed of a data model, which mathematically relates the unknown and known parameters, by a system of equations, and a numerical method to solve that system of equations. Hence, it is clear that different data models can be used along with different numerical methods (in fact in the recent years some works $[11,13-16]$ about this combination have been presented).

For this reason, one of the purposes of this paper is to propose an additional, wider, and fully compatible classification for any localization algorithm. This classification is based on a proposed scheme that defines a localization algorithm as the pair composed by the data model and the numerical method. In the same sense, an aim of this paper, after the analysis of the most representative data model and numerical methods in the open literature, was to provide general conclusions and guidelines for applying them for MLAT surveillance. In fact, following the proposed framework, the efficiency of any localization algorithms can be divided in two components: the first one is the statistical efficiency, which provides the measurement about the bias and variance of the position error distribution, and the second one is the numerical efficiency, which is related to the possibility to provide a valid numerical value (i.e., one obtained with convergence of the solution) for the target position, regardless of the statistical characteristics of it. It is clear that to achieve a suitable statistical efficiency, the numerical one must be ensured. Normally when the DOP [17] of the system presents good (i.e., limited) values, the 
analysis of the different localization algorithms is not critical as most of them approximately reach the theoretical accuracy with the same performance. However, when the DOP either has large (or very large) values or rapidly changes (e.g., like in airport surface because of the closeness of the receiving stations), the overall efficiency of the localization algorithm plays an important role on the system accuracy, which has to be deeply analyzed and characterized.

Several works can be found in the literature regarding the theoretical analysis (i.e., for the analysis of the lower, theoretical bounds of accuracy) of the MLAT systems [7-9], but no general work was found, which analyzes the performance of the various localization algorithms.

Last, to better understand the powerful of the classification proposed, also some real TOA/TDOA data, recorded from the airport surface movement of a vehicle equipped with a "non-transponder device," have been used to test the most representative localization algorithms in the literature. Furthermore, the vehicle was also equipped with a Differential Global Positioning System (DGPS) receiver, thus supplying highly accurate position data, which is taken as the true target position. Thus, the error distributions, for every analyzed algorithm, are obtained. Finally, the general guidelines about the localization algorithms performance are provided.

This paper is organized as follows: The general framework, the data model, and numerical methods descriptions are provided in Sect. 2, while the corresponding simulation and results, and the numerical analysis are shown in Sect. 3. Finally, the conclusions and general guidelines about the performed analysis are provided.

\section{A general scheme for localization algorithms}

In the frame of the localization problem in MLAT systems, as described in Sect. 1, the target position is a set of values that satisfies a set of equations, in the form of Eqs. (2) and (3). Localization algorithms are generally composed of a data model and of a numerical method. The data model explicitly relates the unknown target position to the set of known parameters by constructing a numerical inverse problem. The numerical method aims to solve the resulting inverse problem. Finding a general scheme is useful for understanding all the localization algorithms, to develop a general classification for them, to facilitate an equivalent comparative analysis among them, and to identify the advantages and disadvantages of each of these. An exhaustive literature search and posterior processing have allowed us to select and to compare the most relevant and representative localization algorithms using the scheme proposed in Fig. 2.

A localization algorithm starts by establishing a characteristic equation relating the unknown target position $\boldsymbol{\theta}$, the measurements $\hat{\mathbf{m}}$, the position $\boldsymbol{\vartheta}_{i}$ of the MLAT stations,
Fig. 2 General framework for localization algorithms

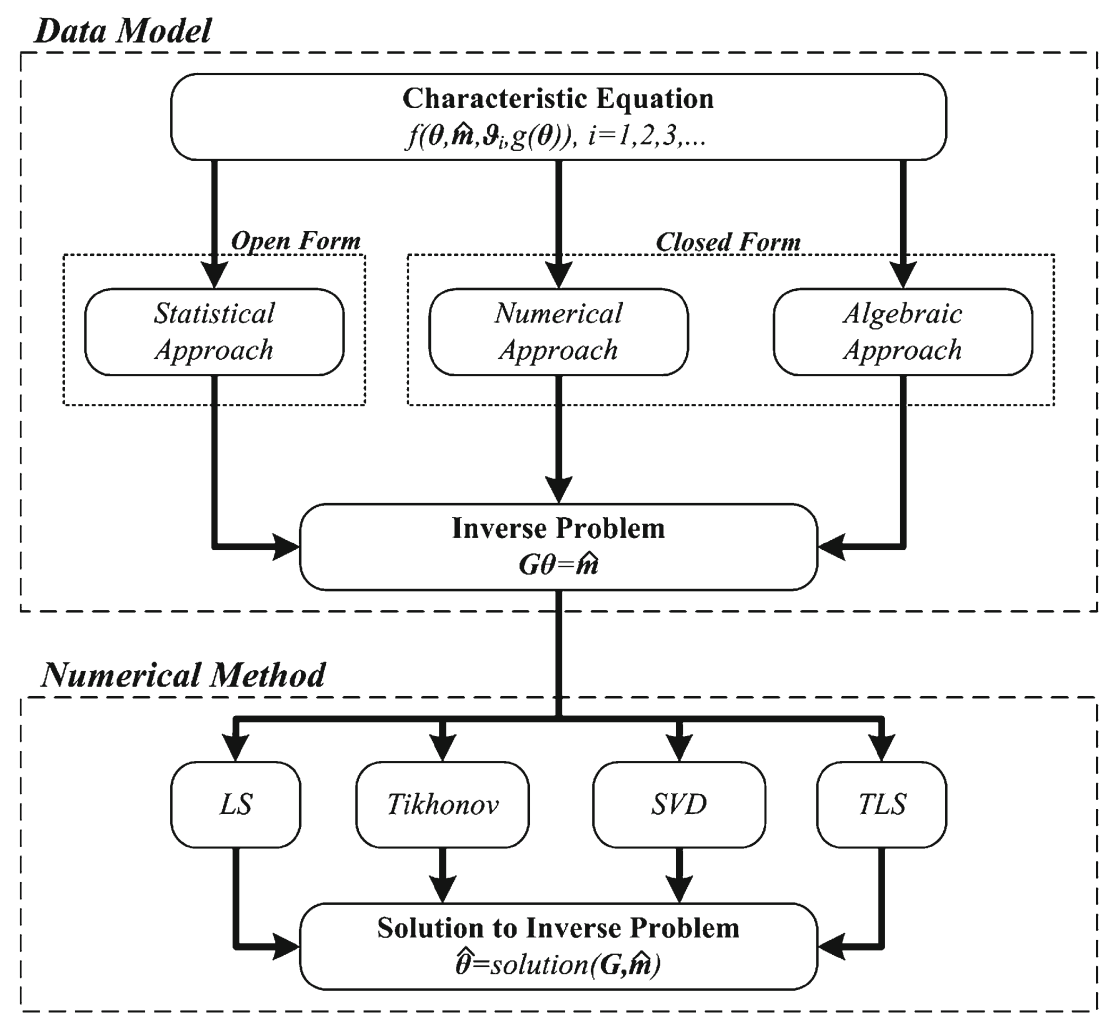


and (optionally) a derived parameter of the target position $\boldsymbol{\theta}$, which is denoted in Fig. 2 as $g(\boldsymbol{\theta})$. Then, the characteristic equation is expressed according to its corresponding data model. Furthermore, the data model is processed, and some approximations, if applicable, are introduced to set up an inverse problem. This inverse problem, as shown in Eq. (3), is generally composed of a coefficient matrix G, which mainly contains the information about the system geometry, of a unknown position vector $\boldsymbol{\theta}$, and a known TDOA measurement vector $\hat{\mathbf{m}}$ (note that, we have introduced the additive noise term into the noiseless measurement vector). Likewise, different pairs of coefficient matrix and measurement vector will result in different localization problems, i.e., every localization algorithm is, in principle, characterized by its own coefficient matrix $\mathbf{G}$ and measurement vector $\hat{\mathbf{m}}$. Finally, the inverse problem can be solved by any of the available numerical methods, and thus, the solution for the target position $\hat{\boldsymbol{\theta}}$ is obtained. A localization strategy includes (1) a coefficient matrix, (2) a measurement vector, and (3) a numerical method. In this sense, the best localization algorithm, for a given scenario, is the one that best fits to the particular conditions of such a scenario with the best choice of the several component of the algorithm.

Every localization algorithm can be classified in this proposed scheme that is more accurate than the classical one that (1) only define a localization algorithm as a unique entity always in the sense of least squares, without taking into account the data model and the numerical algorithms, and (2) only classifies the algorithms only as open and closed form. Both points do not take into account the most relevant characteristics that set the algorithms performance. In this new classification scheme, all the assumptions, the hypothesis, and the performance limitation becomes clear for every particular algorithms.

Furthermore, depending on the localization scenario, the right combination of data model and numerical method can be chosen; for example, in the case of airport surface surveillance, the main constraints [1] are as follows: (1) the high requested accuracy and (2) the bad geometry that produces ill-conditioning of the inverse problem to be solved.

The following subsections describe the most representative data models and numerical methods, their mathematical background, and the different localization algorithms that belong to each of them and to understand their possible performance and limitation.

\subsection{The data model}

The characteristic equation, which was used before but not formally defined, is very useful to explain the proposed classification of any localization algorithm. Basically, a charac- teristic equation relates the unknowns to the measurements collected by a set of receiving stations and their positions. In this sense, when this relationship is transformed into a vector-matrix form [see (3) or Fig. 1], for a set of $N_{s}$ stations, it is called data model. Therefore, the data model represents the basic form for the particular inverse problem as constructed by any localization algorithm. The data models used by the different localization algorithms can be classified as (1) statistical, (2) numerical, and (3) algebraic. In the following, the main characteristics of each of these are described.

\subsubsection{The statistical approach-based models}

This class of models assumes certain statistical hypotheses about the measurements and the target position and a set a probabilistic model that relates to each other. Most of them are based on the maximum likelihood (ML) principle [18] due to the asymptotic consistency and efficiency of the ML estimators (MLE). Concerning the measurement error distributions, Gaussian distributions are generally assumed $[6,7,9]$. To solve this kind of highly nonlinear models, linear approximations and iterative numerical methods are required. Consequently, a reasonable a-priori estimation of the solution is needed and the convergence is not always guaranteed, see [16] for a complete analysis of this problem. The statistical approach algorithms are commonly classified as open-form algorithms. On the other hand, if the statistical hypotheses are satisfied by the measured data, these models provide optimum estimators, i.e., estimators that are in practice unbiased and with covariance matrix very close to its Cramer-Rao Lower Bound (CRLB) [9].

The statistical approach-based models require at least of $n+1$ stations for a $n$-dimensional localization. Moreover, they provide a unique solution and introduce only linear noise terms in the resulting inverse problem. The latter is very important for wide area multilateration (WAM), where the measurement noise due to propagation losses is considerably large [9].

The typical problem that is solved by the localization algorithms using this kind of data models is the maximization of the likelihood function defined as follows:

$$
\begin{aligned}
& \Lambda(\boldsymbol{\theta}) \\
& =\frac{1}{(2 \pi)^{\frac{N_{s}-1}{2}} \operatorname{det}(\mathbf{N}(\boldsymbol{\theta}))^{\frac{1}{2}}} e^{-\frac{1}{2}\left\{(\hat{\mathbf{m}}-\mathbf{m}(\boldsymbol{\theta}))^{T} \mathbf{N}(\boldsymbol{\theta})^{-1}(\hat{\mathbf{m}}-\mathbf{m}(\boldsymbol{\theta}))\right\},}
\end{aligned}
$$

where $\mathbf{m}(\boldsymbol{\theta})$ describes the exact range differences as in Eq. (2) without the random noise term, $\mathbf{N}(\boldsymbol{\theta})$ is the covariance matrix of the measurement errors, and det () denotes the determinant. Thus, the likelihood function is maximized by minimizing the following nonlinear function: 
$Q(\boldsymbol{\theta})=(\hat{\mathbf{m}}-\mathbf{m}(\boldsymbol{\theta}))^{T} \mathbf{N}(\boldsymbol{\theta})^{-1}(\hat{\mathbf{m}}-\mathbf{m}(\boldsymbol{\theta}))$

It is the most general statistical data model considered here. The algorithms to treat with this kind of models commonly use a Taylor series approximation of the function $\mathbf{m}(\boldsymbol{\theta})$ to obtain a linear relation that allows the construction of a linear inverse problem. This linear approximation is generally expressed as follows:

$\mathbf{m}(\boldsymbol{\theta})=\mathbf{m}\left(\boldsymbol{\theta}_{0}\right)+\mathbf{J}\left(\boldsymbol{\theta}-\boldsymbol{\theta}_{0}\right)$,

where $\mathbf{J}$ is the Jacobian differential matrix of $\mathbf{m}(\boldsymbol{\theta})$ (see [7, 11] for details), and $\boldsymbol{\theta}_{0}$ is the required previous estimation of the solution, also called starting point. The convergence of the localization algorithms initially depends on the starting point quality, although the numerical method that is used can work with relatively poor accuracy for the starting points [11].

The most relevant localization algorithms that uses this kind of data models are those described in [19], which was statistically described and analyzed in [7], and the ones proposed in [11] and [16]. The main difference in these algorithms is that the ones in $[7,19]$ set the problem in the sense of least squares (LS), while the one in [11] set the problem in the sense of Tikhonov regularization, and the one in [16] in the sense of the singular value decomposition (SVD)-based regularization. The algorithms proposed in $[11,16]$ allows convergence even in the large DOP values situations.

\subsubsection{The numerical approach-based models}

These models set a mathematical function that relates the unknown target position, the measurements, and a parameter formally derived from the target position (e.g., the target distance from one station). The resulting models are linear in one unknown given the other one. Then, they assume certain numerical approximations between the target position and its derived parameter in order to simplify the solution. The most common assumed approximation is that of mutual independence between them. These numerical approximations are independent of the statistical distributions of the measurement errors. Most of them are based on setting an error function whose squared norm is minimized. These models can be solved by direct optimization and do not require any previous estimation of the solution; for this reason, the algorithms based on them are commonly classified as closedform algorithms. Furthermore, normally, the computational cost required to solve this kind of models is less than that required for the statistical approach-based ones. On the other hand, the solutions provided by them are generally biased and not optimum in the statistical sense.

This kind of data model, depending on the numerical assumptions, can require $n+1$ or $n+2$ receiving stations for $n$-dimensional localization. Moreover, some of them can provide two possible solutions for the target position as they set a quadratic data model. Thus, these ones require an additional procedure to choose one of the two possible solutions. Likewise, this data model always introduces quadratic noise terms in the resulting inverse problem.

The typical error function $\varepsilon$ that is set by this kind of data model can be, in a general sense, expressed as follows:

$\boldsymbol{\varepsilon}=\boldsymbol{\delta}-2 R_{S} \hat{\mathbf{m}}-2 \mathbf{S} \boldsymbol{\theta}$,

where $\delta$ is a known vector that depends on the range difference measurements and on the station positions, $R_{S}$ is the unknown target range with respect to a reference point (see Fig. 1), $\mathbf{S}$ is a known matrix that may depend on both the range difference measurements and on the station positions (i.e., it is the matrix that contains the information about the system geometry), and $\boldsymbol{\theta}$ is the unknown target position. The error function vector $\boldsymbol{\varepsilon}$ in (7) is linear in $R_{S}$ given $\boldsymbol{\theta}$ and vice versa.

The most representative algorithms using this kind of models are the one by Smith and Abel [20], the one by Friedlander [21], the one by Schau and Robinson [22], and the one by Chan an Ho [23]. They are a suitable representation of the most of the algorithms of this class. For example, the Smith and Abel algorithm [20] mathematically solves (7) to obtain $R_{S}$ as a function of $\boldsymbol{\theta}$ and then uses this solution, again into (7), to obtain the corresponding solution for $\boldsymbol{\theta}$. These two solutions are obtained by minimizing the square root of $\boldsymbol{\varepsilon}$. The Friedlander algorithm assumes $\boldsymbol{\varepsilon}=\mathbf{0}$ and multiplies the resulting problem by a matrix that contains the measurement vector $\hat{\mathbf{m}}(\boldsymbol{\theta})$ in its null space, thus eliminating the term $R_{S}$ from (7). The Schau and Robinson algorithm also assumes $\boldsymbol{\varepsilon}=\mathbf{0}$, obtains two possible solutions for $R_{S}$ by using an auxiliary quadratic equation that, in some sense, depends on (7), and finally obtain two possible solutions for $\boldsymbol{\theta}$ by solving (7) in the sense of LS. The Chan and Ho algorithm solves (7) in the same sense of Smith and Abel, but it jointly estimates $R_{S}$ and $\boldsymbol{\theta}$. Due to the dependence of these two parameters, Chan and Ho implement a quadratic correction that updates the squares of the target position components. This algorithm also provides two possible solutions.

\subsubsection{The algebraic approach-based models}

These models use neither statistical assumptions nor numerical approximations, but, rather, they algebraically manipulate the hyperbolic equations (2) to directly set an inverse problem that linearly relates the unknown target position to the known parameters (i.e., the measurements and the station positions). These models are very simple as only geometric relations are used. By contrary, they normally require more stations and introduce quadratic and cubic noise terms into the inverse problem. Like the numerical approach-based models, they do not require any previous estimation of the solution and 
can be solved by direct optimization; hence, the algorithms that use these models are also classified as closed-form algorithms. The solutions provided by them are also biased and not optimum in the statistical sense. For this reason, in the literature, this kind of algorithms is classified into the same group of those ones that use a numerical data model. However, as we will show later, the performance provided by the algebraic data model is better than the one provided by the numerical data models. On the other hand, most of them require the lowest computational resources for solving the resulting inverse problem.

This kind of data model can require $n+1$ or $n+2$ stations for $n$-dimensional localization, and as the numerical approach-based models, they can provide one or two possible solutions for the target location, calling for an additional procedure to choose one of them. An advantage of this kind of data models above to the numerical ones is that because of the lack of numerical assumptions, these models can provide higher accuracies with a smaller number of stations.

These algorithms are computationally the simplest. The typical algebraic data model can be expressed, in general sense, as follows:

$A x+B y+C z=D$

where $(x, y, z)$ are the components of the target position $\boldsymbol{\theta}$, and $A, B, C$, and $D$ are coefficients that depends on the measurements and on the positions of a set of stations. Different set of coefficients $A, B, C$, and $D$ means different localization algorithms. The most relevant algorithms using this data model are the one by Schmidt [24], the one by Geyer and Daskalakis [25], and the one only published in the open license website Wikipedia ${ }^{\circledR}[26]$. Particularly, the Geyer and Daskalakis [25] algorithm is a practical implementation of the Bancroft algorithm [27], which was originally proposed for GPS and that is based on TOA measurements rather than TDOA or range differences. It is the only one that set a data model something different than that of Eq. (8).

\subsection{Numerical methods}

The resulting linear inverse problem $\mathbf{G} \boldsymbol{\theta}=\hat{\mathbf{m}}$ [see (3) or Fig. 1], and obtained by any data model, must be numerically solved. In addition to the fact that the matrix $\mathbf{G}$ and the measurement vector $\hat{\mathbf{m}}$, for every particular localization algorithm, can take a particular form, the numerical efficiency of every particular solution strongly depends on the numerical method used to solve that inverse problem. As commented before, most numerical method suited to solve the resulting linear inverse problem belongs to the least-squares family, i.e., use the pseudoinverse matrix [28]. Moreover, among the models based on the statistical approach (that require iterative procedures because the nonlinearities involved), the GaussNewton method [19] is the most commonly used. However, the latter can be also seen as an iterative solution in the sense of least squares. In the following, the most relevant numerical methods, found in the literature, are shortly described.

The least-squares method solves the following residual error norm minimization problem:

$\hat{\boldsymbol{\theta}}=\arg \min _{\boldsymbol{\theta}}\|\mathbf{G} \boldsymbol{\theta}-\hat{\mathbf{m}}\|^{2}$

The solution $\hat{\boldsymbol{\theta}}$ provided by (9) is known to be the minimum residual norm when solved by the pseudoinverse matrix [28]. However, this solution is not always numerically stable, and hence, it does not always provide acceptable accuracies. As a matter of fact, in many operational conditions, the coefficient matrix $\mathbf{G}$ has some linearly dependent rows, i.e., the corresponding DOP parameter takes large values. This situation has been analyzed in $[9,16]$.

Other robust numerical methods do exist and have been recently implemented for the MLAT localization problem. These algorithms include the Tikhonov regularization, the SVD, and the total least-squares (TLS) regularization. They are intended to provide numerical stable solutions and solve a modified version of the residual error norm in Eq. (9).

The Tikhonov regularization solves a linear combination of the residual error norm in (9) and an auxiliary norm called "smoothed" norm, as follows:

$\hat{\boldsymbol{\theta}}_{\text {Tikhonov }}=\arg \min _{\boldsymbol{\theta}}\left\{\|\mathbf{G} \boldsymbol{\theta}-\hat{\mathbf{m}}\|^{2}+\lambda^{2}\|\mathbf{L} \boldsymbol{\theta}\|^{2}\right\}$,

where $\lambda$ and $\mathbf{L}$ are called regularization parameter and matrix of Tikhonov, respectively, and the term $\|\mathbf{L} \boldsymbol{\theta}\|$ is the smoothed norm. The regularization parameter and the matrix must be previously estimated. The authors in [11] provide and simple, but efficient procedure for that estimation in MLAT localization.

The SVD-based regularization, specifically the Truncated SVD (T-SVD), solves an equivalent minimization problem to (9) but by using a modified (truncated) version of matrix G, as follows:

$\hat{\boldsymbol{\theta}}_{\mathrm{T}-\mathrm{SVD}}=\arg \min _{\boldsymbol{\theta}}\left\|\mathbf{G}_{k} \boldsymbol{\theta}-\hat{\mathbf{m}}\right\|^{2}$,

where $\mathbf{G}_{k}$ is the modified version of $\mathbf{G}$, and $k$ is known as the "truncation parameter." The matrix $\mathbf{G}_{k}$ is obtained by filtering out the $n-k$ last singular values [28] of matrix $\mathbf{G}$, where $n$ is the number of columns of matrix $\mathbf{G}$ (i.e., the number of target coordinates to be estimated). Thus, if the correct truncation parameter $k$ is estimated, then the modified matrix $\mathbf{G}_{k}$ is numerically stable and does not contain linearly dependent rows, allowing a suitable numerical solution to the problem in Eq. (11). A procedure to obtain the truncation parameter and the corresponding modified matrix $\mathbf{G}_{k}$ is described in [16] for MLAT localization.

The TLS-based regularization, specifically the Truncated TLS (T-TLS), assumes that also the coefficient matrix $\mathbf{G}$ is 
perturbed by some errors and, under this assumption, solves the following LS problem:

$$
\begin{aligned}
& \widehat{\boldsymbol{\theta}_{\mathrm{T}}-\mathrm{TLS}} \\
& \quad=\operatorname{argmin}_{\theta}\left\|[\mathbf{G}, \widehat{\mathbf{m}}]_{k_{\mathrm{T}-\mathrm{TLS}}}-\left[\widetilde{\mathbf{G}}, \widehat{\mathbf{m}}^{\prime}\right]\right\|_{F} \text { subject to } \widehat{\mathbf{m}}^{\prime}=\widetilde{\mathbf{G}} \boldsymbol{\theta}
\end{aligned}
$$

where $\widetilde{\mathbf{G}}$ is the perturbed coefficient matrix, $\hat{\mathbf{m}}^{\prime}$ is the equivalent perturbed version of the measurement vector, \|\|$_{F}$ denotes de Frobenius norm of a matrix [28], $k_{\mathrm{T}-\mathrm{TLS}}$ is the corresponding T-TLS truncation parameter, and the matrix $[\mathbf{G}, \hat{\mathbf{m}}]_{k_{\mathrm{T}-\mathrm{TLS}}}$ is a modified version of matrix $[\mathbf{G}, \hat{\mathbf{m}}]$. The notation $[\mathbf{A}, \mathbf{b}]$ means that the vector $\mathbf{b}$ extents the matrix $\mathbf{A}$ to the right in one column. Some particular application of T-TLS method, for some numerical data model-based algorithms, can be found in $[13,14]$. The corresponding procedures depend on some parameters that are not explicitly provided; thus, no equitable comparison can be achieved.

\section{Simulation and results}

In order to help us to compare the various localization algorithms, the company ERA A.S. has provided us with a record of TDOA measurements of one of its operational systems, the MLAT system installed at Tallinn airport (Tallinn, Estonia). This is a particular localization problem for surface surveillance. The system is composed of fourteen receiving stations, and the record of TDOA measurements was taken through the entire airport surface following the requested procedures by the European regulatory bodies [1]. The record contains more than 4000 register (with an average period of $1 \mathrm{~s}$ ), where each register contains set of TDOA measurements. Moreover, the company above mentioned has provided highly accurate position reference data, simultaneously recorded with a GPS receiver with differential correction capabilities (DGPS). These data are used to calculate the 2D error of the various analyzed localization algorithms. The system layout and the reference position data are depicted in Fig. 3. This data set has been chosen because of some critical environmental situations during its collection.
In this scenario, also considering the main constrains cited in Sect. 2, we are looking for statistically (not biased) and numerically (always converging) efficient algorithm. Following the general scheme that we propose, the best choice is the combination of a statistical approach (usually not biased) along with a regularized numerical method (e.g.,Tikhonov or T-SVD), to manage the numerical instability of the solution due to the not suitable geometry conditions.

To validate this statement, we have simulated the localization algorithms by Schmidt [24], Foy [19], Smith and Abel [20], Friedlander [21], Schau and Robinson [22], Chan and Ho [23], the application of Bancroft by Geyer and Daskalakis [25], and the Wikipedia ${ }^{\circledR}$ [26]. All of these algorithms use the least-squares numerical method.

Moreover, we also have simulated the particular applications of Tikhonov and T-SVD regularization methods to the statistical approach proposed by the authors in [11] and [16].

For the localization algorithms using a statistical approach-based model, which require for a starting point, we have used the one provided by the Schau and Robinson algorithm. Moreover, for those algorithms that require choosing one of the two possible solutions (i.e., the Schau and Robinson algorithm, the Chan and Ho, and the Bancroft), we have used the same selection procedure.

To evaluate the accuracy of the above-mentioned algorithms, we followed the indication of the regulatory body, and we have calculated the target position through the entire path depicted in Fig. 3 and obtained, for each of them, the corresponding 2D $(x, y)$ error against the DGPS reference position data. Then, the standard deviation and mean of each error distribution are obtained, as described in [1,2]. These values are shown in Table 1 .

As we can see from Table 1, all the localization algorithms solved in the sense of LS provide very large values of standard deviation and mean, significantly much greater than the requested one for surface surveillance [1]. In this sense, the Schmidt, Friedlander, and Wikipedia algorithms provide the smallest values of standard deviation and mean (385 and $37 \mathrm{~m}$, respectively). Then, the Smith and Abel, Schau and Robinson, and Bancroft algorithms provide greater values.

Fig. 3 Tallinn MLAT system

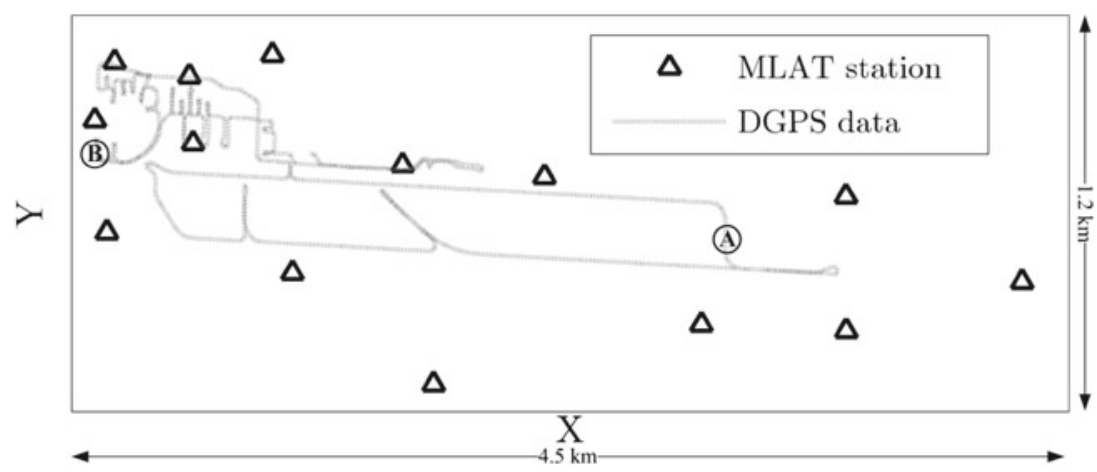


Table 1 Standard deviation and mean for the error distribution of every localization algorithm

\begin{tabular}{llll}
\hline Model & Algorithm & $\sigma(\mathrm{m})$ & Mean $(\mathrm{m})$ \\
\hline A & Schmidt & 385.93 & 39.74 \\
S & Foy & NaN & NaN \\
N & Smith and Abel & 614.66 & 67.16 \\
N & Friedlander & 385.15 & 36.67 \\
N & Schau and Robinson & 616.26 & 108.62 \\
N & Chan and Ho & 737.49 & 876.57 \\
A & Bancroft & 456.06 & 97.61 \\
A & Wikipedia & 384.66 & 35.38 \\
S & Statistical + Tikhonov & 61.79 & 12.57 \\
S & Statistical + T-SVD & 65.03 & 12.75 \\
\hline
\end{tabular}

Values in meters ( $N$ numerical, $S$ statistical, $A$ algebraic). $N a N$ not a number

The Foy algorithm is the one with the greatest error values, providing no useful average performance mainly due to lack of convergence (i.e., NaN: Not a Number), through the entire path. Regarding the Chan and Ho algorithm, which provides the largest value of mean (with the exception of Foy algorithm), the reason is its low capability of jointly obtaining the target range and the target position. In this case, when this algorithm applies the quadratic correction [23], as the target range is highly inaccurate (in some cases negative), this correction also leads to a highly inaccurate positions. We have found that if only the first solution of this algorithm (i.e., before the quadratic correction), for target position, is taken as the final one, it presents an equivalent performance as Smith and Abel algorithm. We do not show these additional results because our objective was to analyze the complete algorithm and its main improvement, which basically is the quadratic correction of the first target position estimation. The main reason for the large values of standard deviation and mean is the conditioning of the localization problem for this scenario, a surface movement scenario with the stations close to each other.

Finally, the localization algorithms solved in the sense of Tikhonov and T-SVD provide the best values of standard deviation and mean. This algorithms use a statistical approach-based models as Foy algorithm does. It is clear how these numerical methods allow the convergence of such statistical model and then, in agreement with the theory, provide the most statistically efficient solutions (minimum standard deviation and mean).

Moreover, as requested in [1], during the measurements recording, the surface vehicle must stop in some particular points of the airport, and measurements are recorded for a certain period. This step permits knowing the general system accuracy, through time, in those points, as several trials are performed. In this paper, we present the results for two of
Table 2 Standard deviation and mean for the error distributions at point A

\begin{tabular}{llll}
\hline Model & Algorithm & $\sigma(\mathrm{m})$ & Mean $(\mathrm{m})$ \\
\hline A & Schmidt & 44.51 & 30.93 \\
S & Foy & 6.7 & 9.4 \\
$\mathrm{~N}$ & Smith and Abel & 61.9 & 14.23 \\
$\mathrm{~N}$ & Friedlander & 21.9 & 20.71 \\
$\mathrm{~N}$ & Schau and Robinson & 335 & 103.19 \\
$\mathrm{~N}$ & Chan and Ho & 839 & 1005 \\
$\mathrm{~A}$ & Bancroft & 178 & 98 \\
$\mathrm{~A}$ & Wikipedia & 22.23 & 21.51 \\
$\mathrm{~S}$ & Statistical + Tikhonov & 5.5 & 7.7 \\
S & Statistical + T-SVD & 4.4 & 6.7 \\
\hline
\end{tabular}

Values in meters ( $N$ numerical, $S$ statistical, $A$ algebraic)

Table 3 Standard deviation and mean for the error distributions at point B

\begin{tabular}{llll}
\hline Model & Algorithm & $\sigma(\mathrm{m})$ & Mean $(\mathrm{m})$ \\
\hline A & Schmidt & 11.06 & 20.10 \\
S & Foy & 6.48 & 12.52 \\
N & Smith and Abel & 15.49 & 18.82 \\
N & Friedlander & 16.01 & 22.71 \\
N & Schau and Robinson & 834 & 194 \\
N & Chan and Ho & 842 & 1135 \\
A & Bancroft & 220.54 & 135.92 \\
A & Wikipedia & 9.94 & 15.16 \\
S & Statistical + Tikhonov & 2.5 & 9.16 \\
S & Statistical + T-SVD & 3.3 & 9.88 \\
\hline
\end{tabular}

Values in meters ( $N$ numerical, $S$ statistical, $A$ algebraic)

these points, which are depicted in Fig. 3 as A and B. The corresponding standard deviations and means are shown in Tables 2 and 3, respectively.

From Tables 2 and 3, we can observe roughly the same ranking of algorithms for these particular analyses. Furthermore, for all the analyzed algorithms, the standard deviation and means are smaller than the corresponding values for the entire path. This analysis as at fixed target position is useful to set some more remarks. First, the algebraic approachbased models (i.e., the Schmidt and Wikipedia algorithms) approximately provide the best accuracy values among the closed-form algorithms (i.e., among the numerical and algebraic approach-based models). This behavior is strongly connected with the fact that they do not make any assumption, nor statistical neither numerical. From the numerical approachbased model algorithms, those that directly provide only one solution (i.e., Smith and Abel, and Friedlander algorithms) also provide acceptable accuracy values, while those ones that provide two possible solutions, i.e., which solve a kind of quadratic problem, are highly dispersed. Then, the statisti- 
cal approach-based models algorithms provide the most statistically efficient solutions for airport surface surveillance. Moreover, for these particular points (A and B), the Tikhonov and T-SVD applications provide the best results among the analyzed algorithms. The reason for this superior performance is that these algorithms have the statistical efficiency of the statistical approach-based models, and the numerical robustness of the regularization techniques, which basically ensure the convergence under a larger number of situations. Note that, for the statistical approach-based models, we use, as the starting point, the solution provided by Schau and Robinson algorithm, which presents large standard deviation an mean values.

Finally, it has to be explained that, in the Central Processing Subsystem (CPS) of any MLAT system, there is always implemented a set of tracking algorithms [29], which significantly reduce the standard deviation of the "rough" output of an MLAT localization algorithm. However, this paper was not aimed to carry out the performance analysis of those tracking algorithms but, rather, to analyze the performance of the sole localization algorithms. In fact, the more accurate the data of these algorithms are, the more accurate the results of the tracking algorithms.

\section{Conclusions}

We have proposed a general scheme to understand and to compare standard and novel $[11,16]$ localization algorithms for MLAT. In this framework, a localization algorithm is characterized by a data model and a numerical method. For the data models, we propose three different approaches that encompass the most of localization algorithms in the literature. This classification is fully compatible with the current one, which only classifies the algorithms as open- or closedform algorithms.

We have tested all the described localization algorithms for a real data scenario. In this application (airport surface surveillance), we have found that the statistically optimal solutions are provided by the algorithms using a statistical approach-based model, as long as the statistical hypotheses are met and the algorithm convergence is reached. However, the convergence of these algorithms when solved in the sense of least squares is not always guaranteed. We have also shown the novel result that the corresponding convergence can be guaranteed when using regularization techniques like Tikhonov or T-SVD, as proposed from the authors. For this application, the first one (Statistical+Tikhonov) shows always the best results.

Concerning the algorithms that use a numerical approachbased model, they are by definition no statistically optimal, but some of them provide better convergence and a low computational cost. However, they may need suit- able geometrical conditions to obtain satisfactory results. Regarding the algorithms that use an algebraic approachbased model, they also are not statistically optimal. However, due to their nature, they provide a stable performance. Moreover, they also need a greater minimum number of stations.

In general, there is no "superior" algorithm that provides the best performance under any scenario or any situation (i.e., from short range, 2D localization to long range, 3D localization, and with different layout). Therefore, a previous analysis of the algorithms performance is always advisable. Likewise, in order to obtain the most efficient localization strategy, it is advisable to use the combination of a statistical approach-based model algorithm (that is, an open-form algorithm) along with an algebraic approach-based model algorithm (a closed-form algorithm).

Acknowledgments Mr. Ivan A. Mantilla-Gaviria has been supported by a FPU scholarship (AP2008-03300) from the Spanish Ministry of Education. Moreover, the authors are grateful to ERA A.S. who supplied the recording of TDOA measurements.

\section{References}

1. EUROCAE-WG-41: ED-117, MOPS for Mode S multilateration systems for use in advanced surface movement guidance and control systems (A-SMGCS). In: The European Organisation for Civil Aviation Equipment (EUROCAE) (November 2003)

2. EUROCAE-WG-70: ED-142, technical specification for wide area multilateration (WAM) systems. In: The European Organisation for Civil Aviation Equipment (EUROCAE) (September 2010)

3. Galati, G., Genderen, P.V.: Proceedings Book of the Tyrrhenian International Workshop on Digital Communications, Enhanced Surveillance of Aircraft and Vehicles (TIWDC/ESAV'11), Capri, Italy (2011)

4. Galati, G., Genderen, P.V.: Proceedings Book of the Tyrrhenian International Workshop on Digital Communications, Enhanced Surveillance of Aircraft and Vehicles (TIWDC/ESAV'08), Capri, Italy (2008)

5. Galati, G., Zellweger, A.: Proceedings Book of the ATM 2002 Advanced Workshop. Capri, Italy (2002)

6. Hahn, W.R., Tretter, S.A.: Optimum processing for delay-vector estimation in passive signal arrays. IEEE Trans. Inf. Theory IT19(5), 608-614 (1973)

7. Torrieri, D.J.: Statistical theory of passive location systems. IEEE Trans. Aerosp. Electron. Syst. AES-20, 183-198 (1984)

8. Levanon, N.: Lowest GDOP in 2-D scenarios. IEE Proc. Radar Sonar Navig. 147(3), 149-155 (2000)

9. Galati, G., Leonardi, M., Mantilla-Gaviria, I.A., Tosti, M.: Lower bounds of accuracy for enhanced mode-s distributed sensor networks. IET Radar Sonar Navig. 6(3), 190-201 (2012). doi:10.1049/ iet-rsn.2011.0197

10. Smith, J.O., Abel, J.S.: Closed-form least-squares source location estimation from range-difference measurements. IEEE Trans. Acoust. Speech Signal Process. ASSP-35(12), 1661-1669 (1987)

11. Mantilla-Gaviria, I.A., Leonardi, M., Galati, G., BalbastreTejedor, J.V., Reyes, E.D.L.: Efficient location strategy for airport surveillance using Mode-S multilateration systems. Int. J. Microw. Wireless Technol. 4(2), 209-216 (2012). doi:10.1017/ S1759078712000104 
12. Romero, L., Mason, J.: Evaluation of direct and iterative methods for overdetermined systems of TOA geolocation equations. IEEE Trans. Aerosp. Electron. Syst. 47(2), 1213-1229 (2011)

13. Yang, K., An, J., Bu, X., Sun, G.: Constrained total least-squares location algorithm using time-difference-of-arrival measurements. IEEE Trans. Veh. Technol. 59(3), 1558-1562 (2010)

14. Weng, Y., Xiao, W., Xie, L.: Total least squares method for robust source localization in sensor networks using TDOA measurements. Int. J. Distrib. Sens. Netw. 2011 (Article ID 172902) (2011). doi:10. 1155/2011/172902

15. Mantilla-Gaviria, I.A., Leonardi, M., Galati, G., Balbastre-T, J.V., Reyes, E.D.L.: Improvement of multilateration (MLAT) accuracy and convergence for airport surveillance. In: Tyrrhenian International Workshop on Digital Communications-Enhanced Surveillance of Aircraft and Vehicles (ESAV'11), Capri, Italy (September 12-14, 2011)

16. Mantilla-Gaviria, I.A., Leonardi, M., Balbastre-Tejedor, J.V., Reyes, Edl: On the application of singular value decomposition and Tikhonov regularization to ill-posed problems in hyperbolic passive location. Math. Comput. Model. 57(7-8), 1999-2008 (2013). doi:10.1016/j.mcm.2012.03.004

17. El-Rabbany, A.: Introduction to GPS: The Global Positioning System, 2nd edn. Artech House, Boston, USA (2006)

18. Trees, HLv: Detection, Estimation and Modulation Theory, Part I. Wiley, New York (2001)

19. Foy, W.H.: Position-location solution by Taylor-series estimation. IEEE Trans. Aerosp. Electron. Syst. AES-12(2), 187-194 (1976)
20. Smith, J.O., Abel, J.S.: The spherical interpolation method of source localization. IEEE J. Ocean. Eng. OE-12(1), 246-252 (1987)

21. Friedlander, B.: A passive localization algorithm and its accuracy analysis. IEEE J. Ocean. Eng. OE-12(1), 234-245 (1987). doi:10. 1109/JOE.1987.1145216

22. Schau, H.C., Robinson, A.Z.: Passive source localization employing intersecting spherical surfaces from time-of-arrival differences. IEEE Trans. Acoust. Speech Signal Process. ASSP-35(8), 1223 1225 (1987)

23. Chan, Y.T., Ho, K.C.: A simple and efficient estimator for hyperbolic location. IEEE Trans. Signal Process. 42(8), 1905-1915 (1994)

24. Schmidt, R.O.: A new approach to geometry of range difference location. IEEE Trans. Aerosp. Electron. Syst. AES-8(6), 821-835 (1972)

25. Geyer, M., Daskalakis, A.: Solving passive multilateration equations using Bancroft's algorithm. In: Digital Avionics Systems Conference, pp. F41/41-F41/48, Bellevue, WA, USA (1998)

26. Wikipedia: http://en.wikipedia.org/wiki/Multilateration

27. Bancroft, S.: An algebraic solution of the GPS equations. IEEE Trans. Aerosp. Electron. Syst. AES-21(7), 56-59 (1985)

28. Golub, G.H., Loan, C.F.V.: Matrix Computations, 3rd edn. The Johns Hopkins University Press, Baltimore (1996)

29. Bar-Shalom, Y., Li, X.R., Kirubarajan, T.: Estimation with Applications to Tracking and Navigation. Wiley, New York (2001) 